\title{
Online News Coverage of COVID-19 Long Haul Symptoms
}

\author{
Corey H. Basch ${ }^{1}$ ( $\cdot$ Eunsun Park ${ }^{2} \cdot$ Betty Kollia $^{2} \cdot$ Nasia Quinones $^{1}$
}

Accepted: 13 November 2021 / Published online: 3 December 2021

(c) The Author(s), under exclusive licence to Springer Science+Business Media, LLC, part of Springer Nature 2021

\begin{abstract}
A number of the people who have recovered from the acute effects of COVID-19 are facing long term sequelae from the infection. As the COVID-19 pandemic is still evolving, so is knowledge of the long-term effects of the virus on patients who still experience symptoms. Clearly, news media play a crucial role in distributing information and this distribution of information can, in turn, influence the actions of the public. The purpose of this study was to describe the content of news coverage of COVID-19 long haul symptoms currently posted on the internet. This study utilized Google News, a news aggregator service, and included the first 100 English language pieces of news. Video content and news article content were coded in depth for information on COVID-19 long haul symptoms. A total of $41 \%$ of news reports mentioned the length of time that the COVID-19 related symptoms persist. The length of time was reported to range from 1 month to more than 1 year. The symptom most commonly mentioned was tiredness or fatigue (74\%), followed by difficulty breathing or shortness of breath (62 cases; 62\%), and difficulty thinking or concentrating (50 cases; 50\%). Other symptoms were mentioned less frequently. There were no statistically significant differences in any of the content including having video, written news reports, or both video and written news reports by source of the news reports based on consumer, professional, or television or internetbased news $(p=.14)$. More complete coverage by online news media of the long-term effects of COVID-19 enhances public awareness of the post-acute syndromes, augments health providers' awareness of the range of chronic COVID-19 effects and the possibility of a second infection, increases the probability of patients' seeking and obtaining the proper care for their symptoms, and contributes to preventive actions for enhancing public health.
\end{abstract}

Keywords Online news $\cdot$ COVID-19 $\cdot$ Long haul symptoms $\cdot$ Health information seeking

\section{Introduction}

The COVID-19 pandemic has caused undeniable difficulty across the world. At the time this paper was written, there have been over 5 million deaths from COVID-19 [1]. COVID-19 remains a public health crisis as variants continue to spread globally. A number of the people who have recovered from the acute effects of the virus are facing long term sequelae from the infection, since the virus may affect any number of organs. As this pandemic is still evolving, so is knowledge of the long-term effects of the virus on patients who still experience symptoms. These patients

Corey H. Basch

baschc@wpunj.edu

1 Department of Public Health, William Paterson University, University Hall, Wayne, NJ 07470, USA

2 Department of Communication Disorders, William Paterson University, Wayne, NJ 07470, USA have been referred to as "long-haulers" [2] and their symptoms may include the consequences of damage to organs such as kidneys, lungs, or skin, as well as muscle atrophy, and psychosocial problems [2, 3]. In terms of neurological symptoms, not-fully-recovered patients have complained of fatigue, headache, brain fog, cognitive problems, insomnia, depression, anxiety, and delirium [2]. Further studies have shown an increase in cerebrovascular events, new-onset psychosis, evidence of central nervous system (CNS) inflammation/encephalopathy, as well as some peripheral nervous system (PNS) symptoms that may persist for several months [4]. Medical researchers are noticing these complications. A systematic review of long term post-COVID-19 syndrome research indicated that the majority of published studies pertained to fatigue and pain, followed by olfactory and neurologic issues, and report pulmonary complications [5]. Of particular interest are the continuing accounts of post-COVID 19 neurologic diseases [6, 7], because even patients who may have been asymptomatic or have had only 
mild-to-moderate COVID-19 and no abnormal MRI findings, may present with complications [8].

Researchers point out that patient advocacy plays a vital role in symptom identification [3]. This is especially true in emerging situations like the COVID-19 pandemic whereby the influx of information is so overwhelming it is referred to as an 'infodemic' [9]. With an overabundance of information, the public is expected to sift through news and determine what is valid and what is 'fake' $[10,11]$. The inability to distinguish between the two or the belief in fake news or conspiracy theories can compromise preventive and health promoting behaviors [12-14]. Further compounding the situation is the motivation of writers to attract attention with dramatic headlines, $[15,16]$, as well as inconsistencies in messaging preferences between public health organizations and the press [17].

Clearly, news media play a crucial role in distributing information and this distribution of information can, in turn, influence the actions of the public. Studies to date have pointed to the abundance of information on social media and in print news on a variety of COVID-19 related topics ranging from general prevention information to vaccination. To date, there is a paucity of research related to coverage of COVID-19 long haul symptoms in news media. Therefore, the purpose of this study was to describe the content of news coverage of COVID-19 long haul symptoms currently posted on the internet.

\section{Methods}

The methods for this study were adapted from a prior study of COVID-19 on on-line news [18]. This study utilized Google News, a news aggregator service, which is one of the most popular online news sites, with an estimated $150,000,000$ unique monthly visitors [19]. Using the Google News function on a cleared browser, articles (including videos and written news articles) were identified using the key words "COVID long-haul symptoms." The search was conducted in May 2021 and included the first 100 English language pieces of news. All URLs with relevant Englishlanguage content were further analyzed. For the purpose of this study, both written news articles and news segments in the form of videos were defined as news clips intended for network news as informative pieces of medical information.

Video content and news article content were coded in depth for COVID-19 long haul symptoms. We aimed to identify the numerous symptoms in adults and children who had previously tested positive for COVID-19 and who were still experiencing symptoms months later. These symptoms are listed in Table 1. Content categories were developed from a reliable fact sheet [20] that detailed different long-haul symptoms, all content was then coded by one researcher (NQ), and a random sample of $10 \%$ was recoded for inter-rater reliability by a second researcher (CHB). Cohen's kappa $(\mathrm{k}=1.0)$ indicated total interrater agreement. Frequencies and percentages for categorical variables were calculated and chi-square test of independence were run to determine differences in types of news report by source of news reports. All calculations were conducted with SPSS version 26 (IBM SPSS, Armonk, NY). This study did not involve human subjects, and was therefore not subject to review by the Institutional Review Board at William Paterson University per their policy.

\section{Results}

The highest number of news reports regarding COVID-19 long-haul symptoms were written reports (81 cases; $81 \%$ ), followed by reports that had both written and video content (18 cases; $18 \%$ ), and one that was a video news report (1 case; $1 \%$ ). In terms of origin of upload, news reports were mostly posted by different sources such as television or internet-based news ( 86 cases; $86 \%$ ) and then professionals such as medical doctors or registered nurses (14 cases; $14 \%$ ).

A total of $41 \%$ of news reports mentioned the length of time that the COVID-19 related symptoms persist. The length of time was reported to range from 1 month to more than 1 year. The news reports included the following COVID-19 long-haul symptoms: tiredness or fatigue (74 cases; 74\%), difficulty breathing or shortness of breath (62 cases; $62 \%$ ), difficulty thinking or concentrating (50 cases; $50 \%$ ), relief after COVID-19 vaccine (48 cases; $48 \%$ ), treatment availability (44 cases; $44 \%$ ), loss of smell or taste (40 cases; $40 \%$ ), headache or sinus pain (38 cases; $38 \%$ ), joint or muscle pain (36 cases; $36 \%$ ), depression or anxiety (31 cases; $31 \%$ ), other related life issues related to long-haul symptoms (27 cases; $27 \%$ ), symptoms that get worse after physical or mental activities (26 cases; $26 \%$ ), chest pain (23 cases; $23 \%$ ), dizziness on standing (21 cases; $21 \%$ ), job loss or inability to work (20 cases; $20 \%$ ), heart palpitations (i.e., fast-beating or pounding heart) (18 cases; 18\%), sleep disturbances (16 cases; 16\%), fear and/or worry (15 cases; $15 \%$ ), fever ( 10 cases, $10 \%$ ), worse symptoms in women ( 10 cases; $10 \%$ ), difficulty getting help ( 9 cases; $9 \%$ ), hair loss ( 7 cases; $7 \%$ ), swelling of different body parts (6 cases; $6 \%$ ), stroke ( 5 cases; $5 \%$ ), post-traumatic stress disorder involving longterm reactions to a very stressful event (4 cases; $4 \%$ ), worse symptoms in older populations (4 cases; 4\%), waves of long-haul symptoms (4 cases; $4 \%$ ), and post-intensive care syndrome (3 cases; $3 \%$ ). A summary is shown in Table 1. There were no statistically significant differences in any of the content including having video, written news reports, or both video and written news reports by source of the news 
Table 1 Number of news stories reporting on long-haul COVID19 symptoms

\begin{tabular}{|c|c|c|}
\hline Contents & Number of stories & $\begin{array}{l}\text { Percent } \\
(\%) \text { of } \\
\text { total }\end{array}$ \\
\hline Tiredness or fatigue & 74 & 74 \\
\hline Difficulty breathing or shortness of breath & 62 & 62 \\
\hline Difficulty thinking or concentrating & 50 & 50 \\
\hline Relief after COVID-19 vaccine & 48 & 48 \\
\hline Treatment availability & 44 & 44 \\
\hline Loss of smell or taste & 40 & 40 \\
\hline Headache or sinus pain & 38 & 38 \\
\hline Joint or muscle pain & 36 & 36 \\
\hline Depression or anxiety & 31 & 31 \\
\hline Other related life issues related to long-haul symptoms & 27 & 27 \\
\hline Symptoms that get worse after physical or mental activities & 26 & 26 \\
\hline Chest pain & 23 & 23 \\
\hline Dizziness on standing & 21 & 21 \\
\hline Job loss or inability to work & 20 & 20 \\
\hline Heart palpitations & 18 & 18 \\
\hline Sleep disturbances & 16 & 16 \\
\hline Fear and/or worry & 15 & 15 \\
\hline Fever & 10 & 10 \\
\hline Worse symptoms in women & 10 & 10 \\
\hline Difficulty getting help & 9 & 9 \\
\hline Hair loss & 7 & 7 \\
\hline Swelling of different body parts & 6 & 6 \\
\hline Stroke & 5 & 5 \\
\hline $\begin{array}{l}\text { Post-traumatic stress disorders involving long-term reactions to a very } \\
\text { stressful event }\end{array}$ & 4 & 4 \\
\hline Worse symptoms in older populations & 4 & 4 \\
\hline Waves of long-haul symptoms & 4 & 4 \\
\hline Post-intensive care syndrome & 3 & 3 \\
\hline
\end{tabular}

${ }^{\mathrm{a}}$ Based on an $\mathrm{n}=100$, contents are not mutually exclusive reports based on consumer, professional, or television or internet-based news $(p=.14)$.

\section{Discussion}

Several long-term effects of COVID-19 infection are currently being reported by healthcare professionals. Patients who have survived the disease but have not recovered fully are seen to exhibit a wide array of symptoms affecting a large number of systems. News coverage in written or video format, by professionals, consumers, or television/internet news, all point out the various physical and mental ill-effects of the virus on the human body.

This has further significance as the number of patients with COVID-19 increases, thus increasing the number of those patients who might exhibit long COVID-19. Certain of these long-haul diseases (e.g., stroke, encephalitis) may result in permanent disability, with its concomitant care needs and socioeconomic costs [21]. For healthcare professionals, recognizing long haul symptoms in their patients may result in timely implementation of effective management techniques to improve those symptoms. For example, Baig warns that patients with long haul COVID-19 who are experiencing neuro-COVID may face permanent disability with all the socioeconomic consequences that it entails [22].

Further research is necessary to determine the role that media plays in describing long-haul COVID-19 symptoms. In a study of the social media platform Reddit, researchers analyzed narrative posts and concluded that social media and digital technologies have played a pivotal role in identifying symptoms and have the ability to influence recovery efforts, particularly in an emerging situation such as the COVID19 pandemic [23]. With proper information, public health officials, healthcare providers, educators, and employers and patients themselves may be able to recognize symptoms consistent with long COVID. The findings of this study indicate that several potential symptoms are not often mentioned in 
online news. Professional healthcare associations' websites should prioritize information including links to sites with reliable facts and comprehensive information.

More complete coverage by online news media of the long-term effects of COVID-19 enhances public awareness of the post-acute syndromes, augments health providers' awareness of the range of chronic COVID-19 effects and the possibility of a second infection, increases the probability of patients' seeking and obtaining the proper care for their symptoms, and contributes to preventive actions for enhancing public health.

\section{Acknowledgements None.}

Author Contributions $\mathrm{CH}$ Basch and B Kollia conceptualized the study. NQ collected the data, EP conducted the data analysis. All authors contributed to the manuscript production.

\section{Funding None.}

\section{Declarations}

Conflict of interest All authors declare that they have no competing interest.

Ethical Approval This study did not involve human subjects, and was therefore not subject to review by the Institutional Review Board at William Paterson University per their policy.

Guarantor All.

\section{References}

1. World Health Organization. Coronavirus disease (COVID-19) pandemic. Retrieved November 29, 2020, from https://www. who.int/emergencies/diseases/novel-coronavirus-2019.

2. Scordo, K. A., Richmond, M. M., \& Munro, N. (2021). PostCOVID-19 syndrome: Theoretical basis, identification, and management. AACN Advanced Critical Care, 32, 188-194.

3. Nalbandian, A., Sehgal, K., Gupta, A., Madhavan, M. V., McGroder, C., Stevens, J. S., Cook, J. R., Nordvig, A. S., Shalev, D., Sehrawat, T. S., Ahluwalia, N., Bikdeli, B., Dietz, D., Der-Nigoghossian, C., Liyanage-Don, N., Rosner, G. F., Bernstein, E. J., Mohan, S., Beckley, A. A., ... Wan, E. Y. (2021). Post-acute COVID-19 syndrome. Nature Medicine. https://doi. org/10.1038/s41591-021-01283-z

4. Varatharaj, A., Thomas, N., Ellul, M. A., et al. (2020). Neurological and neuropsychiatric complications of COVID-19 in 153 patients: A UK-wide surveillance study. Lancet Psychiatry, 7, 875-882.

5. Salamanna, F., Veronesi, F., Martini, L., Landini, M. P., \& Fini, M. (2021). Post-COVID-19 syndrome: The persistent symptoms at the post-viral stage of the disease. A systematic review of the current data. Frontiers in Medicine, 8, 653516.

6. Ellul, M. A., Benjamin, L., Singh, B., Lant, S., Michael, B. D., Easton, A., et al. (2020). Neurological associations of COVID19. Lancet Neurology, 19, 767-783.

7. Abrams, R. M. C., Kim, B. D., Markantone, D. M., Reilly, K., Paniz-Mondolfi, A. E., Gitman, M. R., et al. (2020). Severe rapidly progressive Guillain-Barre syndrome in the setting of acute COVID-19 disease. Journal of Neurovirology, 26, 797-799.

8. Abrams, R. M. C., Safavi, F., Tuhrim, S., Navis, A., Steinberger, J., Shin, S. C. (2021). MRI negative myelopathy post mild SARS-CoV-2 infection: Vasculopathy or inflammatory myelitis? Journal of NeuroVirology

9. World Health Organisation. (2020). Managing the COVID19 infodemic: Promoting healthy behaviours and mitigating the harm from misinformation and disinformation. Retrieved August 30, 2021, form https://www.who.int/news/item/2309-2020-managing-the-covid-19-infodemic-promoting-healt hy-behaviours-and-mitigating-the-harm-from-misinformationand-disinformation

10. Barua, Z., Barua, S., Aktar, S., Kabir, N., \& Li, M. (2020). Effects of misinformation on COVID-19 individual responses and recommendations for resilience of disastrous consequences of misinformation. Progress in Disaster Science, 8, 7-8.

11. Romer, D., \& Jamieson, K. H. (2021). Patterns of media use, strength of belief in COVID-19 conspiracy theories, and the prevention of COVID-19 from March to July 2020 in the United States: Survey Study. Journal of Medical Internet Research, 23, e25215.

12. Allington, D., Duffy, B., Wessely, S., Dhavan, N., \& Rubin, J. (2020). Health-protective behaviour, social media usage and conspiracy belief during the COVID-19 public health emergency. Psychological Medicine. https://doi.org/10.1017/S0033 29172000224X

13. Gerts, D., Shelley, C. D., Parikh, N., Pitts, T., Watson Ross, C., Fairchild, G., Vaquera Chavez, N. Y., \& Daughton, A. R. (2021). "Thought I'd Share First" and other conspiracy theory tweets from the COVID-19 infodemic: Exploratory study. JMIR Public Health and Surveillance, 7, e26527.

14. van Prooijen, J. W., \& van Vugt, M. (2018). Conspiracy theories: Evolved functions and psychological mechanisms. Perspectives on Psychological Science. https://doi.org/10.1177/ 1745691618774270

15. Mock, J. 'Dreck,' Drama: How U.S. Media Handled the Pandemic. Retrieved August 30, 2021, from https://www.webmd. com/lung/news/20210623/dreck-drama-how_us_media_handl ed_the_pandemic.

16. Nielsen, K., Cherubini, F., Andi, S. (2020). Few winners, many losers: the COVID-19 pandemic's dramatic and unequal impact on independent news media. Retrieved August 30, 2021, from https://reutersinstitute.politics.ox.ac.uk/few-winners-many-losers-covid-19-pandemics-dramatic-and-unequal-impact-indep endent-news-media.

17. Ophir, Y. (2018). Coverage of epidemics in American Newspapers through the lens of the crisis and emergency risk communication framework. Health Security.

18. Basch, C. H., Hillyer, G. C., Meleo-Erwin, Z., Mohlman, J., Cosgrove, A., \& Quinones, N. (2020). News Coverage of the COVID-19 Pandemic: Missed opportunities to promote health sustaining behaviors. Infection, Disease, \& Health, 11(3), 205-209.

19. eBizMBA. The eBusiness Guide. Retrieved August 30, 2021, from http://www.ebizmba.com/articles/news-websites.

20. Centers for Disease Control and Prevention. Post-COVID Conditions. Retrieved August 30, 2021, from https://www.cdc.gov/ coronavirus/2019-ncov/long-term-effects.html.

21. World Health Organization (WHO). Understanding and managing Long COVID requires a patient-led approach. Retrieved August 30, 2021, from https://www.euro.who.int/en/ health-topics/health-emergencies/pages/news/news/2021/02/ new-policy-brief-calls-on-decision-makers-to-support-patie 
nts-as-1-in-10-report-symptoms-of-long-covid/understandingand-managing-long-covid-requires-a-patient-led-approach.

22. Baig, A. M. (2020). Deleterious outcomes in long-hauler COVID-19: The effects of SARS-CoV-2 on the CNS in chronic COVID syndrome. ACS Chemical Neuroscience, 11, 4017-4020.

23. Southwick, L., Guntuku, S. C., Klinger, E. V., Pelullo, A., McCalpin, H., \& Merchant, R. M. (2020). The role of digital health technologies in COVID-19 surveillance and recovery: A specific case of long haulers. International Review of Psychiatry. https:// doi.org/10.1080/09540261.2020.1854195

Publisher's Note Springer Nature remains neutral with regard to jurisdictional claims in published maps and institutional affiliations. 